\title{
Total thoracoscopic sleeve lobectomy of the middle and lower lobes of the right lung
}

\author{
Desong Yang, Yong Zhou, Wenxiang Wang \\ The Second Department of Thoracic Surgery, Hunan Cancer Hospital \& The Affiliated Cancer Hospital of Xiangya School of Medicine, Central \\ South University, Changsha, China \\ Correspondence to: Wenxiang Wang. The Second Department of Thoracic Surgery, Hunan Cancer Hospital \& The Affiliated Cancer Hospital of \\ Xiangya School of Medicine, Central South University, Changsha 410013, China. Email: wangwenxiang@hnca.org.cn.
}

\begin{abstract}
This is a video recording of total thoracoscopic sleeve lobectomy performed on a patient with central lung cancer in the lower lobe of the right lung involving the orifice of the bronchus intermedius. The patient, a 50-year-old male, was admitted to the hospital due to cough lasting more than 2 months. The patient had no history of underlying diseases. On admission, the patient had decreased breath sounds in the right lower lobe without significant rales. Chest computed tomography showed a space-occupying lesion in the lower part of the right hilum, atelectasis of the middle and lower lobes of the right lung, and right hilar lymph node enlargement; however, the lymph nodes in the mediastinum were not significantly enlarged. Bronchoscopic examination revealed a neoplasm completely obstructing the orifice of the right bronchus intermedius, which pathological biopsy confirmed to be squamous cell carcinoma. A complete examination showed no evidence of distant metastasis; therefore, total thoracoscopic sleeve lobectomy of the right middle and lower lobes was performed. The 3-port approach was used for the surgery, which was performed through the anterior main operating port. Systematic lymph node dissection was performed first, followed by anatomical resection of the middle and lower lobes of the right lung. The arterial and venous branches of the middle and lower lobes of the right lung were cut, after which the right bronchus intermedius was skeletonized, and the orifice of the right bronchus intermedius was cut. Then, sleeve lobectomy of the orifice of the bronchus intermedius was performed, and finally, 3-0 Prolene sutures were used for continuous suture (from right principle bronchus to right upper lobe bronchus). The operation went smoothly. The patient's postoperative pathological stage was pT2aN0M0 (stage $\mathrm{Ib}$ ).
\end{abstract}

Keywords: Total thoracoscopy; middle and lower lobes of the right lung; sleeve lobectomy

Received: 05 April 2020; Accepted: 12 January 2021; Published: 25 November 2021.

doi: $10.21037 /$ ccts-20-79

View this article at: http://dx.doi.org/10.21037/ccts-20-79

\section{Introduction}

The past 20 years have seen great progress in minimally invasive thoracic surgical techniques. Although minimally invasive surgery has long been used in the treatment of central lung cancer, its development has been slow. Since Santambrogio et al. (1) first reported thoracoscopic bronchial sleeve lobectomy in 2002, this surgical technique has not been rapidly or widely adopted and remains immature. Furthermore, thoracoscopic sleeve lobectomy of the middle and lower lobes of the right lung has yet to be reported in the literature.

The patient in the present study was a 50-year-old male whose bronchoscopic examination revealed a neoplasm completely obstructing the orifice of the right bronchus intermedius; squamous cell carcinoma was confirmed by pathological biopsy. Subsequently, the patient underwent total thoracoscopic sleeve lobectomy of the right middle and lower lobes of the lung using the 3-port approach, and the operation went smoothly. All procedures performed in this study were in accordance with the ethical standards of the institutional and/or national research committee(s), 


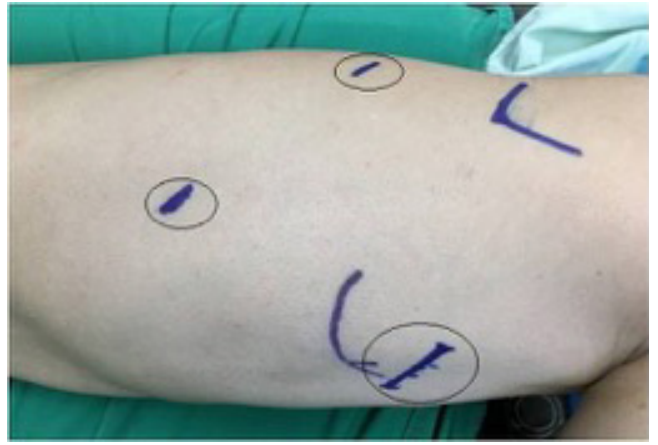

Figure 1 Port placement.

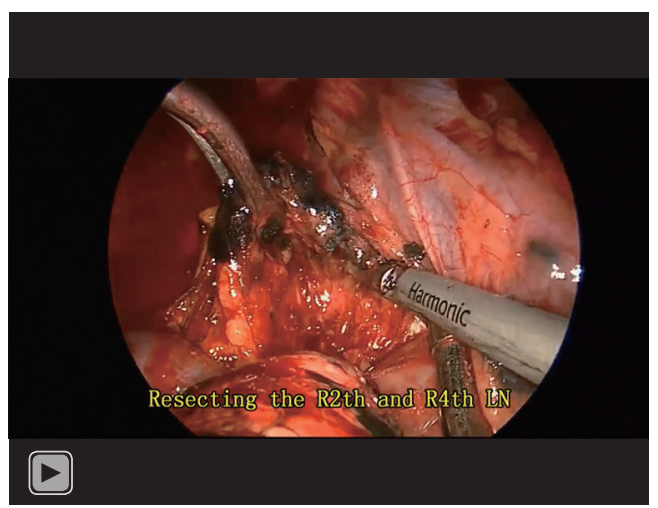

Video 1 Video-assisted thoracoscopic surgery right middle and lower sleeve lobectomy.

and with the Helsinki Declaration (as revised in 2013). Written informed consent was obtained from the patient for publication of this study and accompanying images. A copy of the written consent is available for review by the editorial office of this journal.

\section{Surgical technique}

The patient was put under general anesthesia with a double-lumen endotracheal tube inserted into left principal bronchus. With the patient placed in the left lateral decubitus position, the procedure was performed using the 3-port approach. An observation port with a length of approximately $1 \mathrm{~cm}$ was placed at the $7^{\text {th }}$ intercostal space on the midaxillary line, a main operating port with a length of approximately $3 \mathrm{~cm}$ was created at the $4^{\text {th }}$ intercostal space on the anterior axillary line, and an assisting port with a length of approximately $1 \mathrm{~cm}$ was created at the $7^{\text {th }}$ intercostal space on the right subscapular line (Figure 1).
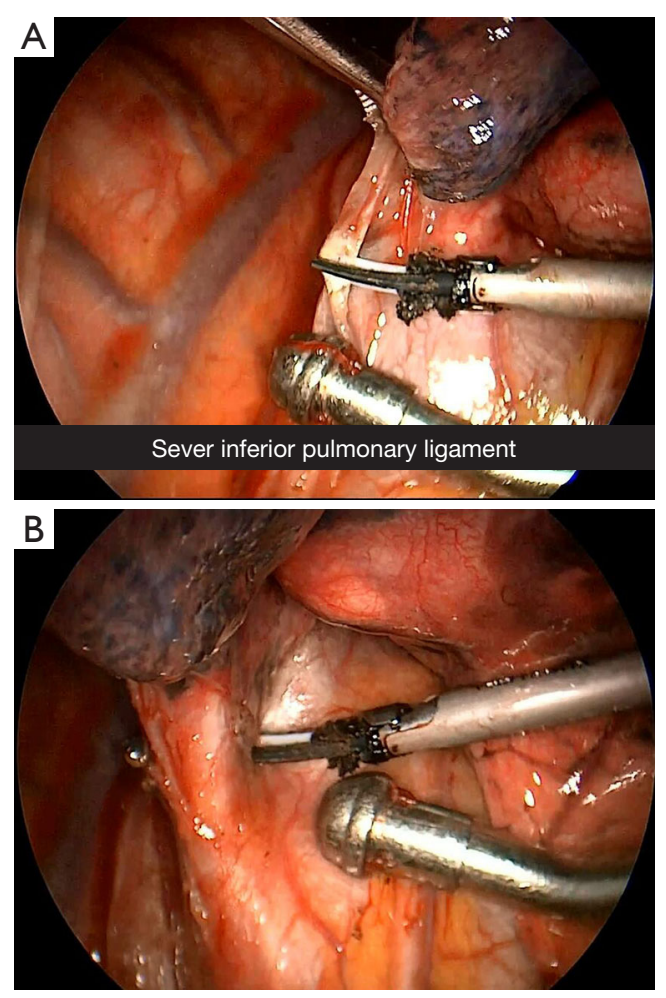

Figure 2 Inferior pulmonary ligament region. (A) Dissection of the inferior pulmonary ligament; (B) dissection completed.

The main operating port was protected with a soft tissue retractor with a diameter of $8 \mathrm{~cm}$. Thoracoscopic sleeve lobectomy of the middle and lower lobes of the right lung was successfully completed using STROZ thoracoscopic equipment (Video 1).

Endoscopic exploration was performed. After careful removal of the thoracic adhesions, systematic mediastinal lymph node dissection was performed in a clockwise direction from bottom to top. This was followed by anatomical resection of the right middle and lower lobes, sleeve lobectomy of the right bronchus intermedius, and anastomosis. Based on the modular surgical procedures promoted by our center, the surgical procedures were divided into 7 modules performed in the following order: (I) inferior pulmonary ligament region; (II) subcarinal region; (III) interlobar region; (IV) superior mediastinal region; (V) hilar and lobar region; (VI) bronchial sleeve resection; (VII) bronchial anastomosis (2). These modules are described below.

Inferior pulmonary ligament region (Figure 2): the right lower lung was pulled upwards, and the inferior pulmonary ligament was cut to the level of the lower edge of the right 

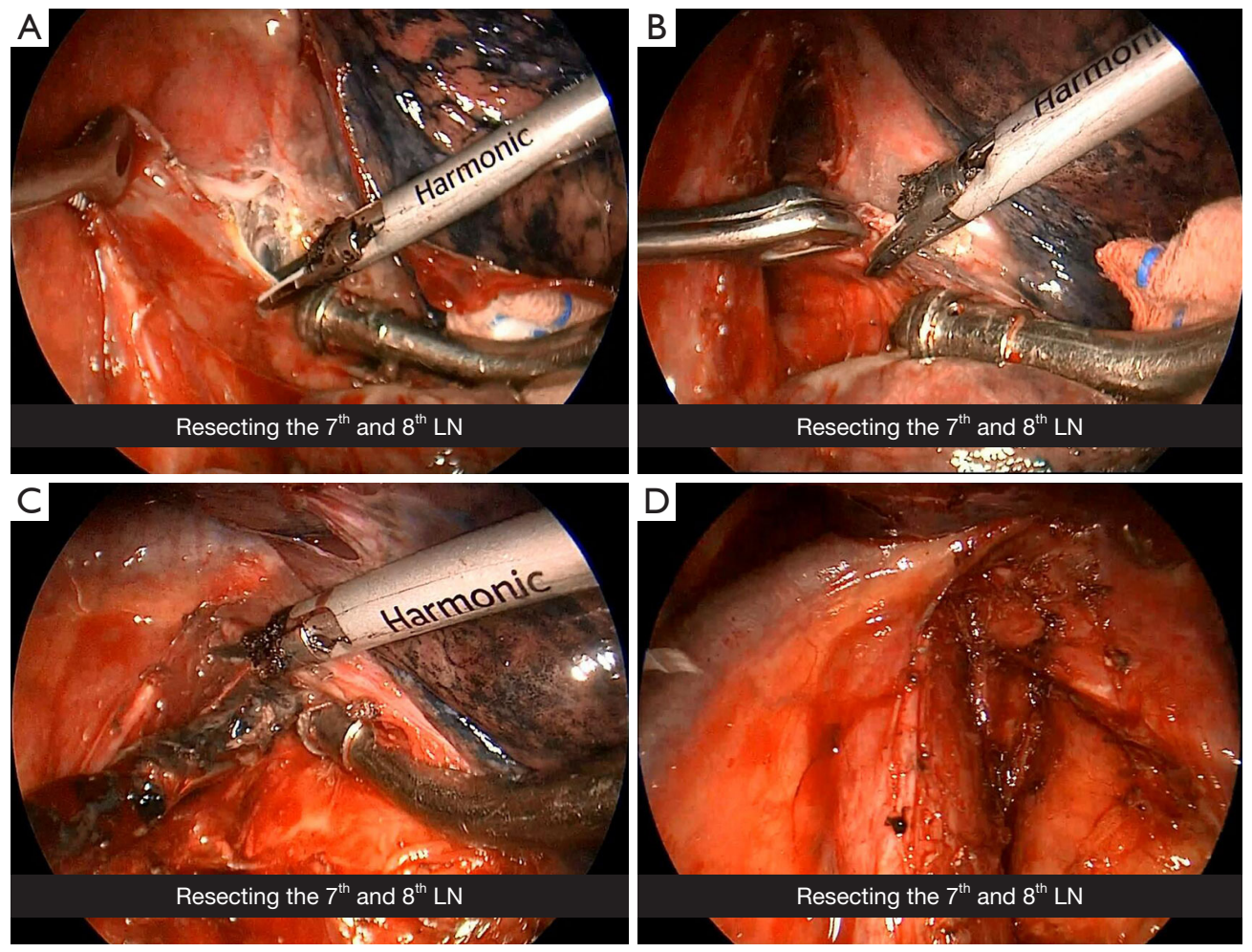

Figure 3 Subcarinal region. (A) Dissection of station 7 and 8 lymph nodes adjacent to the esophagus; (B) dissection of station 7 and 8 lymph nodes along the right principal bronchus; (C) dissection of station 7 and 8 lymph nodes reached the level of the carinal apex; (D) dissection completed. LN, lymph node.

inferior pulmonary vein. Meanwhile, together with the lymph nodes in the inferior pulmonary ligament (group 9) was pushed toward the distal end of the right lower lung.

The subcarinal region (Figure 3): the right lower lung lobe was pulled anteriorly to expose the posterior mediastinum, and the mediastinal pleura in the subcarinal region was incised longitudinally upward in front of the esophagus. The dissection started adjacent to the esophagus and continued until the left principal bronchus was exposed in the deep surface of the subcarinal region and the esophagus was exposed posteriorly. Then, dissection was performed along the right principal bronchus toward the carina, to the level of the carinal apex. Finally, the perforating branch of the bronchial artery was transected at the level of the carinal apex, and the subcarinal and paraesophageal lymph nodes were completely dissected (groups 7 and 8).

The interlobar region (intersection of the right upper lobe/bronchus intermedius, Figure 4): the right upper lobe was pulled toward the top of the chest, and the right middle and lower lobes were pulled down toward the diaphragm to expose the crest of the right upper lobe/bronchus intermedius. An ultrasonic scalpel was used to dissect the region from the side of the right bronchus intermedius toward the crest. If sufficient loosening between the lymph nodes and the adjacent posterior bronchus was observed, the lymph nodes in the region were not dissected at this point.

The superior mediastinal region (Figure 5): the upper lobe of the right lung was pulled downward to expose the upper mediastinum. First, the mediastinal pleura was incised under the arch of the azygos vein, which was pulled upward to expose the area below it, and the adhesion between the lymph nodes in this area and the surrounding area was sufficiently released. Then, an inverted T-shaped incision was made above the arch of the azygos vein and in front of the superior vena cava to open the upper part of mediastinal pleura. First, the lymph nodes were released from the associated tissues laterally and superiorly along the superior vena cava. Next, the lymphoid tissues in this area were pulled up to allow downward incision to the dissection site below. Then, the lymph nodes in this area were pulled 

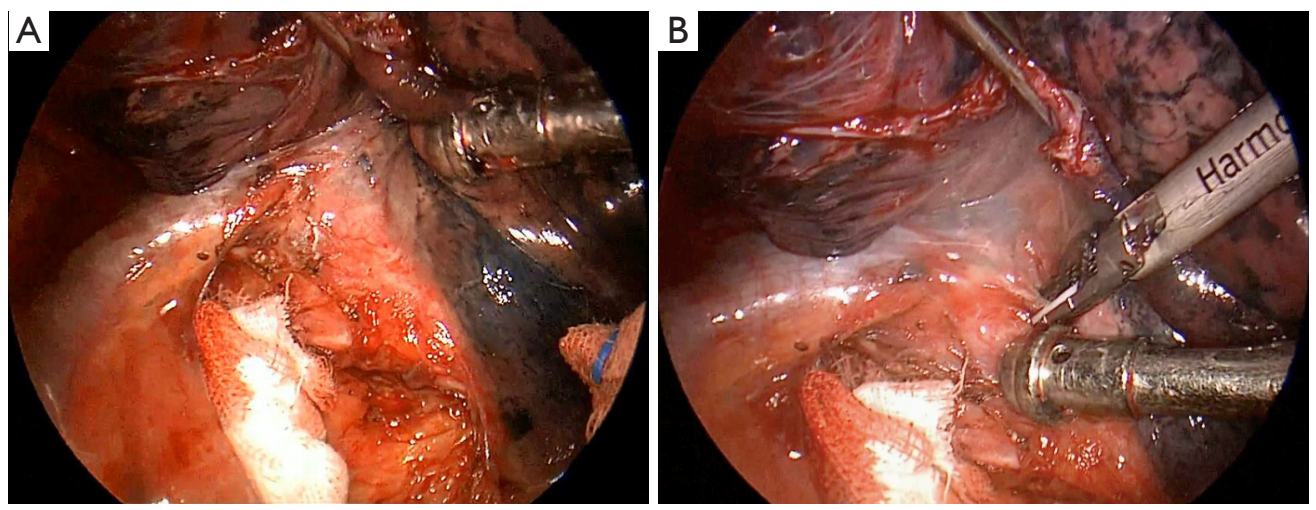

Figure 4 Intersection of the right upper lobe and the bronchus intermedius. (A) Exposed the crest of the right upper lobe/bronchus intermedius; (B) loosening the lymph nodes between right upper lobe bronchus and right intermediate bronchus.
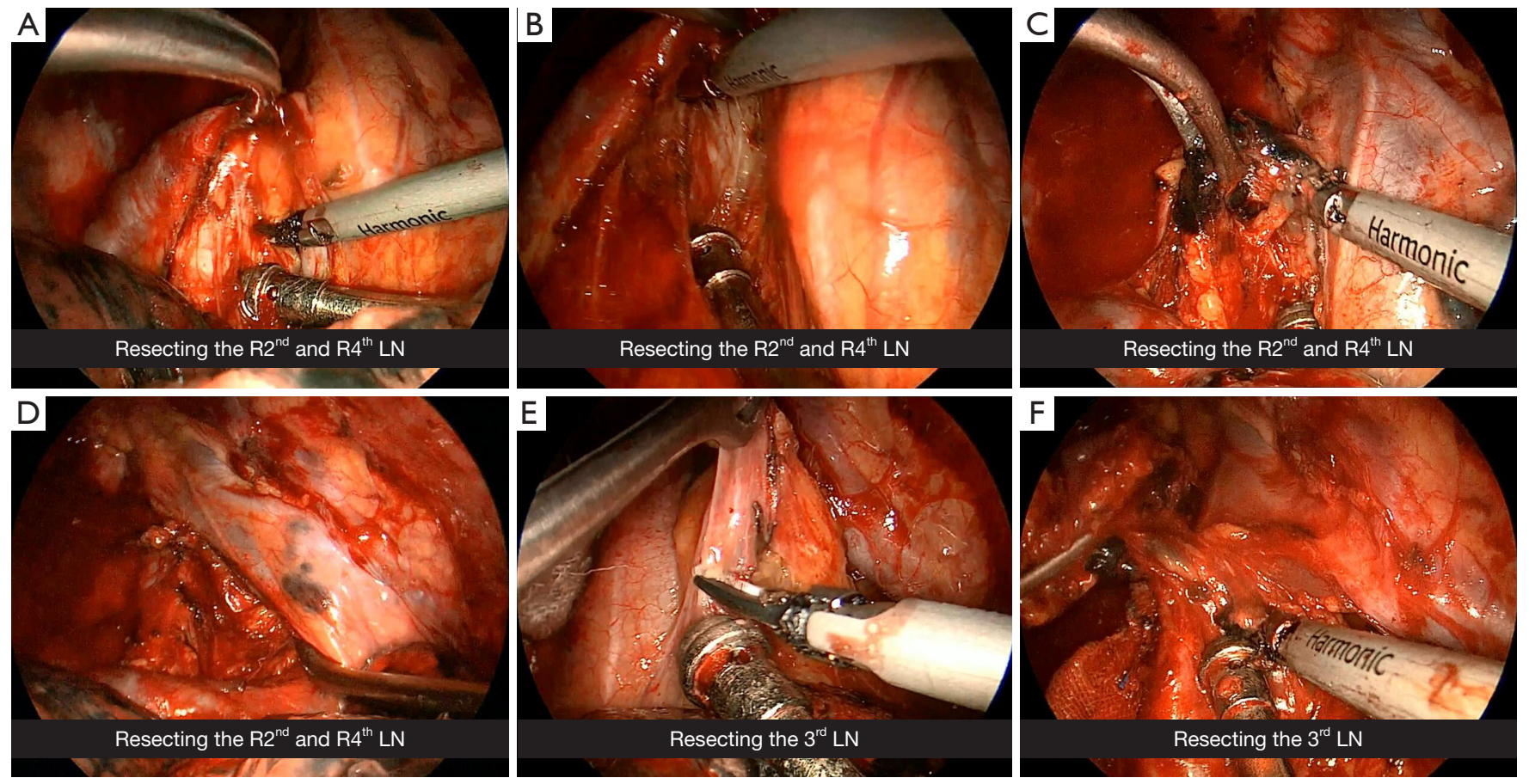

Figure 5 Superior mediastinal region. (A) Incising of the mediastinal pleura under the arch of the azygos vein; (B) dissection of station 2 and 4 lymph nodes under the arch of the azygos vein; (C) dissection of station 2 and 4 lymph nodes over the arch of the azygos vein; (D) dissection of station 2 and 4 lymph nodes completed; (E) dissection of station 3a lymph nodes; (F) dissection of station 3a lymph nodes completed. LN, lymph node.

upward, and the adhesions next to the trachea and the deep pericardial side were continuously released from bottom to top until lymph node groups 2 and 4 in this area were completely dissected. Dissection of lymph node group 3 was continued. The mediastinal pleura was incised from bottom to top, starting in front of the origin of the superior vena cava outside the pericardium and continuing to the level of the lower edge of the left innominate vein. The lymph nodes in front of the blood vessels in this region were removed completely.

Hilar and lobar regions (Figure 6): first, the interlobar adhesion was opened at the intersection of the horizontal 

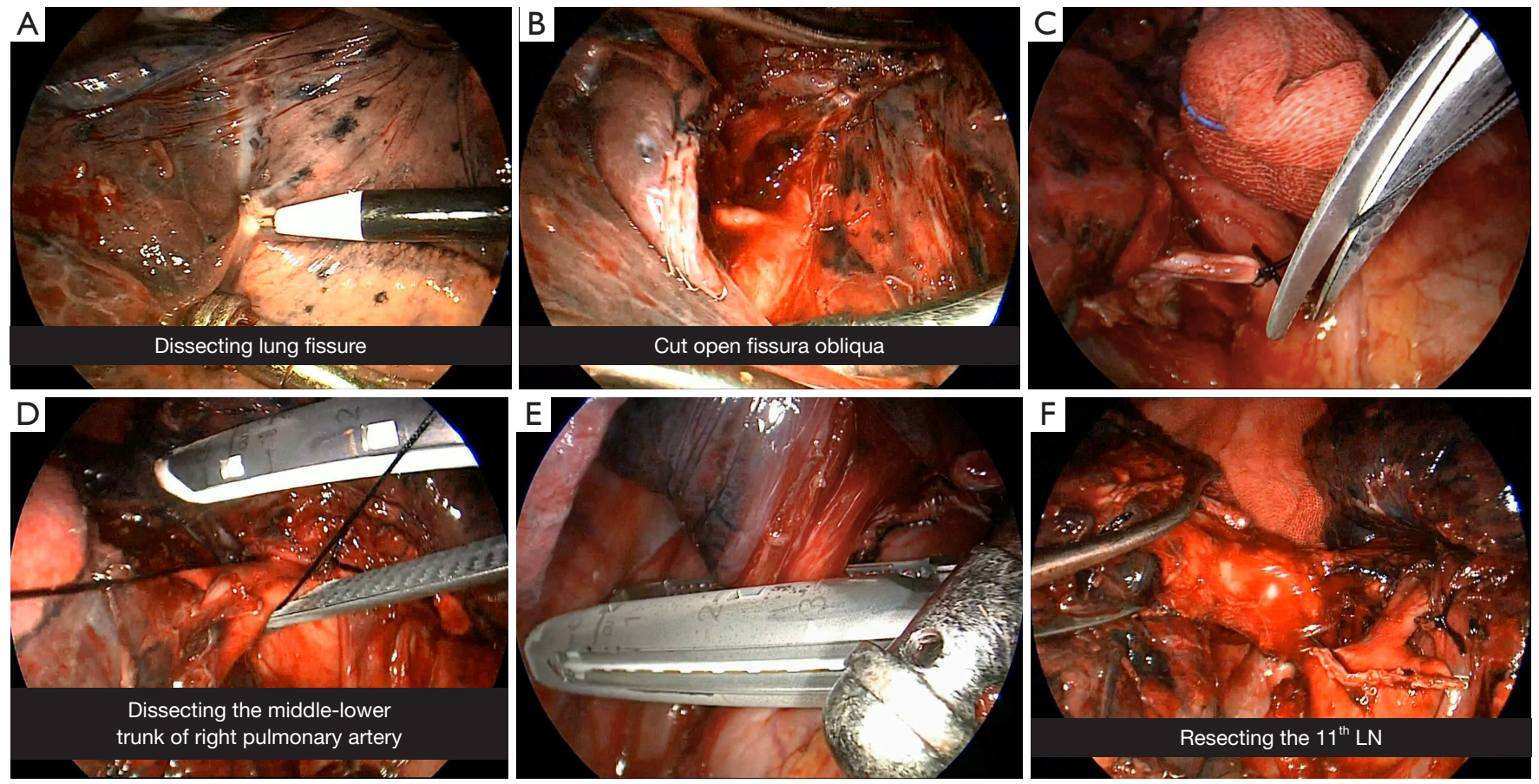

Figure 6 Hilar and lobar regions. (A) Dissection of the interlobar adhesion at the intersection of the horizontal fissure and the oblique fissure; (B) dissection of the lung fissure; (C) dissection of the right middle pulmonary vein; (D) dissection of the right middle-lower pulmonary artery trunk; (E) dissection of the right inferior pulmonary vein; (F) dissection of the station 11 lymph nodes. LN, lymph node.

fissure and the oblique fissure for dissection toward the deep pulmonary trunk. After exposure of the interlobar pulmonary trunk, the vascular sheath on the surface of the pulmonary trunk was carefully opened. The dorsal segmental artery of the right lower lobe was exposed through posterior dissection inside the vessel sheath. After that, the posterior oblique fissure tunnel could be safely opened along the distal end of the dorsal segmental artery and the upper edge of the right bronchus intermedius, and was incised using a disposable linear cutter-stapler with a golden cartridge (Touchstone). Then, forward dissection of the pulmonary trunk inside the vessel sheath was continued between the lobes until the proximal end of the medial segmental branch of the right middle lobe artery was exposed. Dissection continued in front of the hilum; the mediastinal pleura was opened in front of the lower hilum, and the right middle pulmonary vein was released inside the vessel sheath. After double ligation with No. 7 and No. 4 suture thread, the distal end was ligated and cut off. After exposure of the deep pulmonary artery trunk in front of the hilum and penetration of the lobes, the horizontal fissure was closed and cut off using a disposable cutter-stapler with a golden cartridge (Touchstone). The interlobar vein which originated from the superior pulmonary vein running toward the right middle lobe was transected. Dissection of the interlobar pulmonary artery trunk was continued. After sufficient dissection inside the sheath, the interlobar pulmonary artery trunk, right middle lobe artery, dorsal segment of the right lower lobe, and basal segment of the artery trunk were closed and transected using a disposable linear cutter-stapler with a white cartridge (Touchstone). Then, the right middle and lower lobes were lifted, the mediastinal pleura above the right inferior pulmonary vein was incised until the vein was loosened enough, and the right inferior pulmonary vein was transected using a disposable linear cutter-stapler with a white cartridge (Touchstone). The middle and lower lobes of the right lung were pulled aside, the lymph nodes around the root of the right bronchus intermedius were dissected, and the right bronchus intermedius was resected at its origin using scissors. The gross specimens were removed.

Bronchial sleeve resection (Figure 7): the stump of the right bronchus intermedius was lifted, and the proximal end of the right upper lobe bronchus and distal end of 

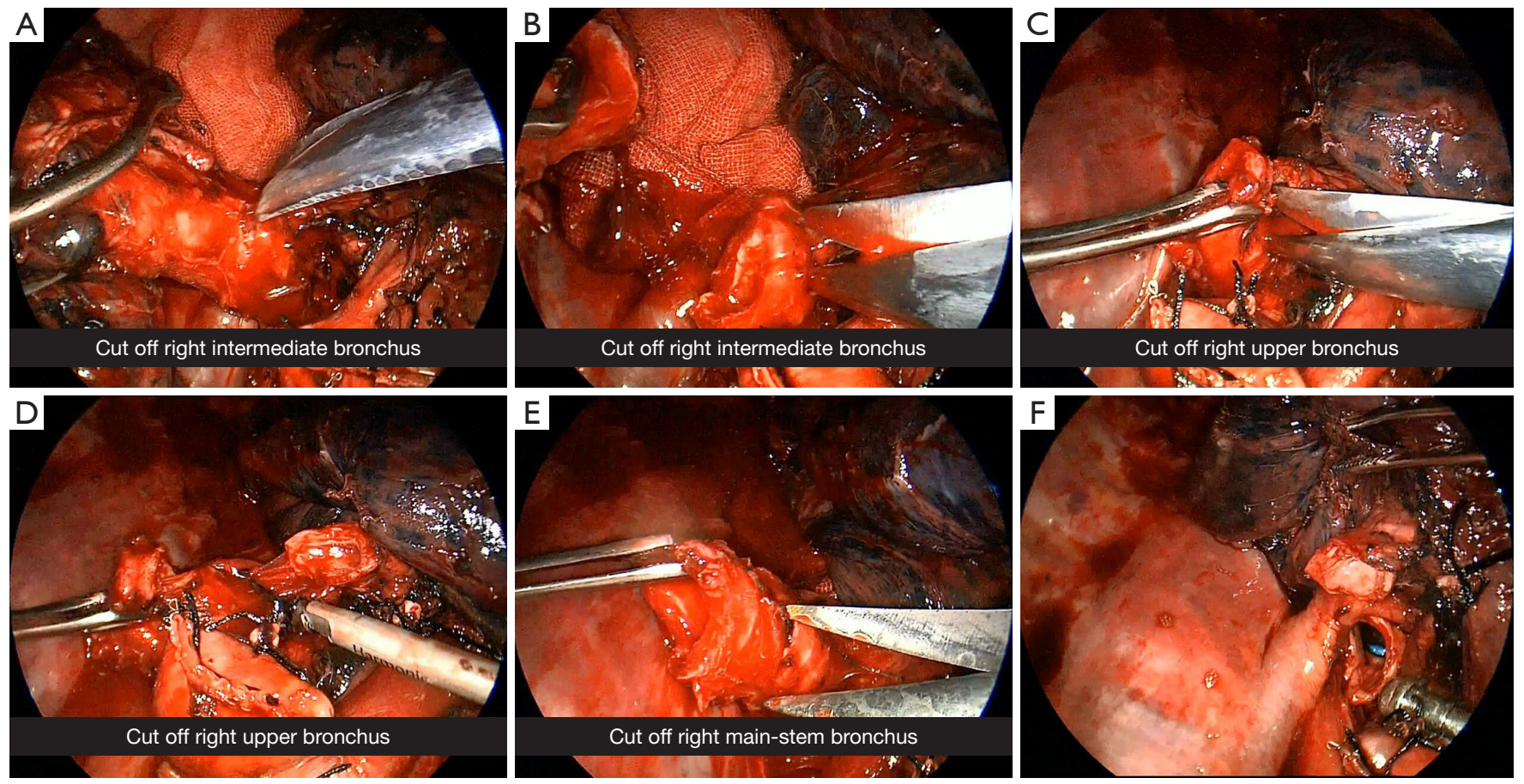

Figure 7 Bronchial resection. (A) Dissection of the right intermediate bronchus; (B) dissection of the right upper lobe bronchus; (C) dissection of the right upper lobe bronchus continued; (D) dissection of the right upper lobe bronchus completed; (E) dissection of the right principal bronchus; (F) dissection completed.

the right principal bronchus were dissected to a hollowed state. Then, the right upper lobe bronchus was resected at its origin with endoscopic scissors. After that, the stump of the bronchus intermedius was lifted, the right principal bronchus was resected and the bronchial stump was trimmed. The proximal and distal resection margins of the bronchi were rapidly frozen for pathological examination. After confirmation that cancerous changes were absent from the resection margins, the sleeve resection was completed.

Bronchial suture (Figure 8): after the distal and proximal resection margins were confirmed negative, bronchial anastomosis was performed using a single-thread, doubleneedle continuous suture method with 3-0 Prolene sutures (Ethicon). The first stitch of the suture was made at the junction of the stump membrane of the right principal bronchus and the cartilage by moving the needle from the inside to the outside. The second stitch was made at the junction of the stump membrane of the right upper lobe bronchus and the cartilage using the other needle, which was also moved gently in an inside-to-outside direction. At this point, no knot was made. Then, the second needle was used to continue the suture of the medial wall from front to back. The outside-to-inside method was used for the stump of the right principal bronchus, and the inside-to-outside method was used for the stump of the right upper lobe bronchus. The suture was gradually tightened and continued to the level of the lower edge of the arch of the azygos vein. Then, the second needle was pulled outward from the main operating port, pulled straight, and fixed in vitro for later use. The first needle in the assisting port was used to continue the suture of the lateral wall from front to back using a continuous suture method via the main operating port until the junction of the two needles was reached. Then, both ends of the suture thread were tightened and knotted. At this point, the continuous suture of the bronchus was complete. A small amount of normal saline was injected into the thoracic cavity to soak the anastomosis. The anesthesiologist was asked to inflate the lung at an airway pressure of $30 \mathrm{cmH}_{2} \mathrm{O}$ to allow for the evaluation of the anastomosis status. No anastomotic leakage was observed.

The operation took 225 minutes to perform and went smoothly. The intraoperative blood loss was $200 \mathrm{~mL}$. The drainage tube was removed on postoperative day 6 , and the patient was discharged on day 7 . The patient's postoperative 

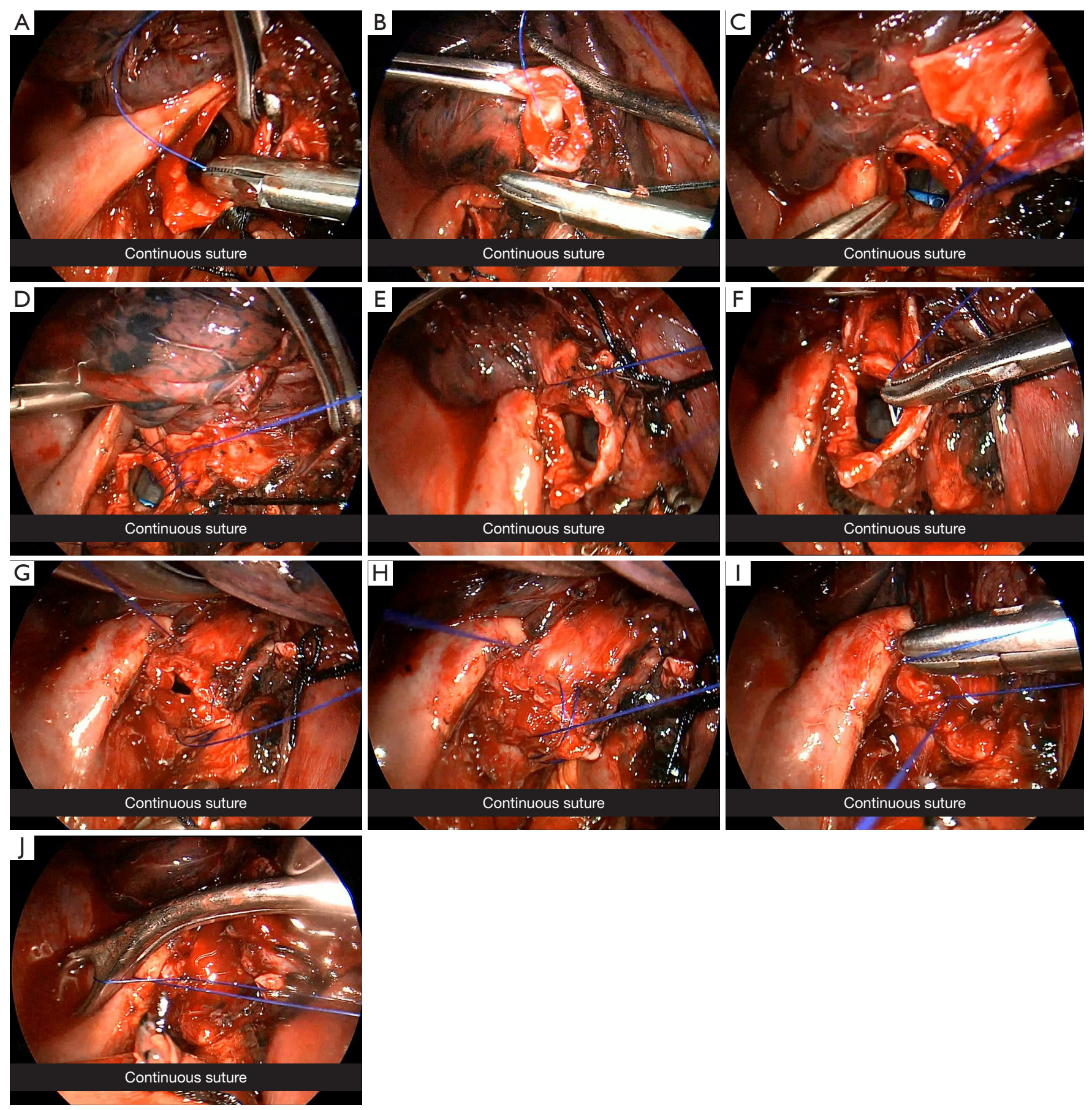

Figure 8 Bronchial anastomosis. (A) The first stitch at the stump of the right principal bronchus from the inside to the outside; (B) the second stitch at the stump of the right upper lobe bronchus from the inside to the outside; $(\mathrm{C})$ continuous suture of the medial wall from front to back; (D) continuous suture of the medial wall; (E) continuous suture of the medial wall completed; (F) continuous suture of the anterolateral wall; $(\mathrm{G})$ continuous suture of the lateral wall from front to back; $(\mathrm{H})$ continuous suture of the lateral wall; (I) the last stitch the continuous suture; (J) continuous suture completed. 
pathological stage was pT2aN0M0 (stage Ib), and R0 resection was achieved.

\section{Comments}

Total thoracoscopic sleeve lobectomy is challenging, because it involves the dissection of hilar structures invaded by the tumor and bronchial anastomosis. Thoracoscopic sleeve lobectomy of the middle and lower lobes of the right lung is a new challenge, but we successfully completed this procedure utilizing our previous experience of approximately 120 cases of thoracoscopic sleeve lobectomy. To rapidly and insightfully perform this difficult surgical procedure, consideration should be given to the following aspects: first, the surgeon must have rich previous thoracoscopic surgery experience so that they can skillfully perform thoracoscopic lobectomy, and they also need to have technical experience in thoracoscopic bronchial $\mathrm{V}$-shaped resection and anastomosis (3). Secondly, systematic lymph node dissection should be performed first. Following dissection of the hilar and mediastinal lymph nodes, the skeletonization of the target-bronchus to be resected can be achieved; this significantly reduces the difficulty of anatomical dissection of the hilum, reduces the risk of the bronchial artery bleeding into the lobes to be preserved after bronchial dissection, and avoids the adverse effects on bronchial anastomosis due to pulling the residual lung if lymph node dissection performed last. Thirdly, a well-developed and reliable bronchial anastomosis technique is critical. At present, continuous suture is the most commonly used technique for bronchial anastomosis (4-6). The 3-0 Prolene suture (single-thread, double-needle) used at our center can significantly reduce suture interference and winding problems during the anastomosis procedure, thus making anastomosis safe, easy, and reliable (2). Based on our experience, we have identified the following key points that can assist in preventing entanglement: (I) continuous suturing with a single suture (single-thread, double-needle) can ensure that only a single suture is required around the anastomosis, which might avoid entanglement caused by several sutures. (II) Gradually converging but not tightening up the suture could make the next entry point easier to locate and help to determine the appropriate needle direction. It could also make it more convenient to stretch the bronchial stump, thereby exposing the entry point. (III) Pulling the other end of the suture from the operation port and maintaining the tension or twining it with a gauze and placing the twined suture and gauze in the thorax could further reduce interference by the suture. (IV) Entanglement could be effectively avoided by inserting the needle with the distant end of the suture over the proximal end to the bronchial stump and then withdrawing the needle below the proximal end of the suture. Fourthly, good team work and thoracoscopic equipment is necessary. Intraoperative use of a double-lumen endotracheal tube, for instance, can provide better exposure of the anastomotic field than the use of a single-lumen tube combined with a bronchial blocker, while proper traction of the bronchial wall provided by an assistant can obtain a better angle for needle insertion. Further, effective cooperation between the thoracoscope operator and the nursing team, as well as the improvement and optimization of double-joint thoracoscopic instruments, can also greatly reduce the difficulty of surgery.

\section{Acknowledgments}

The video was awarded third prize in the First International Lung Surgery Competition (2019 Masters of Lung Surgery).

Funding: The study was supported by Natural Science Foundation for the Youth of Hunan province of China (No. 2018JJ3314 to DY), and Scientific research project of Hunan Health Commission (No. B2019094 to DY).

\section{Footnote}

Peer Review File: Available at https://ccts.amegroups.com/ article/view/10.21037/ccts-20-79/prf

Conflicts of Interest: All authors have completed the ICMJE uniform disclosure form (available at https://ccts. amegroups.com/article/view/10.21037/ccts-20-79/coif). DY reports grants from Natural Science Foundation for the Youth of Hunan province of China (No. 2018JJ3314), grants from Scientific research project of Hunan Health Commission (B2019094), during the conduct of the study. The other authors have no conflicts of interest to declare.

Ethical Statement: The authors are accountable for all aspects of the work in ensuring that questions related to the accuracy or integrity of any part of the work are appropriately investigated and resolved. All procedures performed in this study were in accordance with the ethical standards of the institutional and/or national research 
committee(s), and with the Helsinki Declaration (as revised in 2013). Written informed consent was obtained from the patient for publication of this study and accompanying images. A copy of the written consent is available for review by the editorial office of this journal.

Open Access Statement: This is an Open Access article distributed in accordance with the Creative Commons Attribution-NonCommercial-NoDerivs 4.0 International License (CC BY-NC-ND 4.0), which permits the noncommercial replication and distribution of the article with the strict proviso that no changes or edits are made and the original work is properly cited (including links to both the formal publication through the relevant DOI and the license). See: https://creativecommons.org/licenses/by-nc-nd/4.0/.

\section{References}

1. Santambrogio L, Cioffi U, De Simone M, et al. Videoassisted sleeve lobectomy for mucoepidermoid carcinoma

doi: $10.21037 /$ ccts-20-79

Cite this article as: Yang D, Zhou Y, Wang W. Total thoracoscopic sleeve lobectomy of the middle and lower lobes of the right lung. Curr Chall Thorac Surg 2021;3:39. of the left lower lobar bronchus: a case report. Chest 2002;121:635-6.

2. Yang D, Zhou Y, Wang W. Total thoracoscopic highposition sleeve lobectomy of the right upper lobe of the lung. J Thorac Dis 2018;10:4490-7.

3. Park SY, Lee HS, Jang HJ, et al. Wedge bronchoplastic lobectomy for non-small cell lung cancer as an alternative to sleeve lobectomy. J Thorac Cardiovasc Surg 2012;143:825-831.e3.

4. Han Y, Zhou S, Yu D, et al. Video-assisted thoracic surgery (VATS) left upper sleeve lobectomy with partial pulmonary artery resection. J Thorac Dis 2013;5 Suppl 3:S301-3.

5. Liu K, Jin C, Tian H, et al. Total video-assisted thoracic surgery sleeve lobectomy: suture by both hands. Thorac Cardiovasc Surg Rep 2013;2:43-5.

6. Huang J, Li S, Hao Z, et al. Complete video-assisted thoracoscopic surgery (VATS) bronchial sleeve lobectomy. J Thorac Dis 2016;8:553-74. 